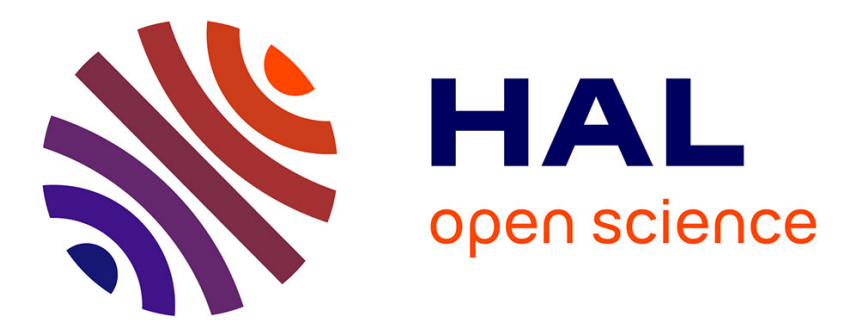

\title{
Retrieving the effective parameters of metamaterials from the single interface scattering problem
}

Jianji Yang, Christophe Sauvan, T. Paul, C. Rockstuhl, F. Lederer, Philippe Lalanne

\section{- To cite this version:}

Jianji Yang, Christophe Sauvan, T. Paul, C. Rockstuhl, F. Lederer, et al.. Retrieving the effective parameters of metamaterials from the single interface scattering problem. Applied Physics Letters, 2010, 97 (6), pp.061102. 10.1063/1.3478241 . hal-00570656

\section{HAL Id: hal-00570656 \\ https://hal-iogs.archives-ouvertes.fr/hal-00570656}

Submitted on 5 Apr 2012

HAL is a multi-disciplinary open access archive for the deposit and dissemination of scientific research documents, whether they are published or not. The documents may come from teaching and research institutions in France or abroad, or from public or private research centers.
L'archive ouverte pluridisciplinaire HAL, est destinée au dépôt et à la diffusion de documents scientifiques de niveau recherche, publiés ou non, émanant des établissements d'enseignement et de recherche français ou étrangers, des laboratoires publics ou privés. 


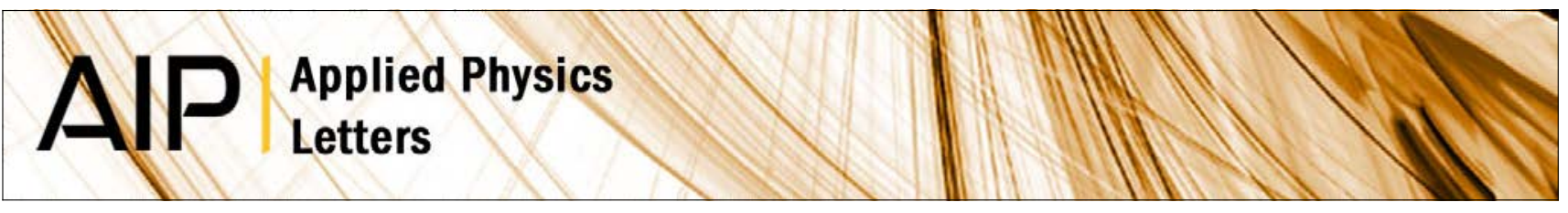

\section{Retrieving the effective parameters of metamaterials from the single interface scattering problem}

J. Yang, C. Sauvan, T. Paul, C. Rockstuhl, F. Lederer et al.

Citation: Appl. Phys. Lett. 97, 061102 (2010); doi: 10.1063/1.3478241

View online: http://dx.doi.org/10.1063/1.3478241

View Table of Contents: http://apl.aip.org/resource/1/APPLAB/v97/i6

Published by the American Institute of Physics.

\section{Related Articles}

Switching nonlinearity in a superconductor-enhanced metamaterial Appl. Phys. Lett. 100, 121906 (2012)

Strong terahertz absorption using $\mathrm{SiO} 2 / \mathrm{Al}$ based metamaterial structures Appl. Phys. Lett. 100, 111104 (2012)

Analysis and experimental demonstration of an active acoustic metamaterial cell J. Appl. Phys. 111, 044505 (2012)

Acoustic metamaterial exhibiting four different sign combinations of density and modulus J. Appl. Phys. 111, 023504 (2012)

Tunable semiconductor metamaterials based on quantum cascade laser layout assisted by strong magnetic field J. Appl. Phys. 110, 123704 (2011)

\section{Additional information on Appl. Phys. Lett.}

Journal Homepage: http://apl.aip.org/

Journal Information: http://apl.aip.org/about/about_the_journal

Top downloads: http://apl.aip.org/features/most_downloaded

Information for Authors: http://apl.aip.org/authors

\section{ADVERTISEMENT}

\section{NEW!}

iPeerReview AIP's Newest App
Authors...

Reviewers...

Check the status of

submitted papers remotely!

ADPublishing 


\title{
Retrieving the effective parameters of metamaterials from the single interface scattering problem
}

\author{
J. Yang, ${ }^{1}$ C. Sauvan, ${ }^{1}$ T. Paul, ${ }^{2}$ C. Rockstuhl, ${ }^{2}$ F. Lederer, ${ }^{2}$ and P. Lalanne ${ }^{1, a)}$ \\ ${ }^{1}$ Laboratoire Charles Fabry de l'Institut d'Optique, CNRS, Univ. Paris-Sud, Campus Polytechnique, \\ RD 128, 91127 Palaiseau, France \\ ${ }^{2}$ Institute of Solid State Theory and Condensed Matter Optics, Friedrich-Schiller-Universität Jena, \\ Max-Wien-Platz 1, 07743 Jena, Germany
}

(Received 7 May 2010; accepted 21 July 2010; published online 9 August 2010)

\begin{abstract}
In order to better understand when man-made structures may be considered as metamaterials with effective properties at optical frequencies, we study the scattering (reflection, transmission) of light at a single interface between a uniform half-space and a bulk metamaterial, i.e., a semi-infinite three-dimensional periodic structure. We accurately compute the scattering coefficients between plane waves and Bloch modes and test the validity of the single-Bloch-mode approximation within the metamaterial. The knowledge of the single-interface scattering coefficients allows us to propose an innovative method for deriving effective optical parameters. (C) 2010 American Institute of Physics. [doi:10.1063/1.3478241]
\end{abstract}

Since the first realization of negative-refractive-index metamaterials (MMs) for microwaves, ${ }^{1}$ intensive work has been performed on this promising topic for ultimately controlling the electric and magnetic components of electromagnetic waves. ${ }^{2,3}$ As the quest for MMs has been moving toward the optical spectral range, various theoretical issues have received considerable attention. The homogenization of artificially structured materials and the computation of their effective properties is one of them. ${ }^{4-12}$ Indeed, because the characteristic length of the functional unit is only slightly smaller than the wavelength, i.e., the MM is mesoscopic, the homogenization of optical MMs is challenging ${ }^{5,6}$ and various numerical techniques have been developed, including field averaging, ${ }^{7}$ Bloch mode approaches, ${ }^{8-10}$ multipole expansion, ${ }^{11,12}$ and inversion of scattering parameters (S-parameters). ${ }^{4,5}$ The latter consists of comparing the scattered waves from a MM slab, which is inherently inhomogeneous, to those scattered from a hypothetical homogeneous slab with both an effective permittivity $\varepsilon_{\text {eff }}$ and permeability $\mu_{\text {eff. }}$ The effective properties are thus derived from calculations or measurements of the S-parameters for finitethickness samples. Owing to its generality that makes it applicable to virtually any structure, the S-parameter retrieval method has rapidly become the most commonly used tool for characterizing artificially structured MMs. But the generality of the method has a downside; since only the far-field response of the MM matters, the retrieval procedure fully ignores the near-field response and the actual light propagation inside the structure.

In order to better understand when man-made structures may be homogenized, we study the scattering of light at a single interface between a uniform half-space (air) and a semi-infinite three-dimensional (3D) periodic structure (the bulk MM), see Fig. 1(a). Taking advantage of an efficient fully-vectorial numerical method, we show that the knowledge of the single-interface scattering coefficients allows for an in-depth test of the validity of the MM homogenization and we propose an innovative retrieval approach that relies

${ }^{a)}$ Electronic mail: philippe.lalanne@institutoptique.fr. on physical quantities that are attached to the fine structure of the bulk MM.

For the sake of illustration, we consider hereafter the fishnet structure composed of rectangular air holes etched in an $\mathrm{Ag}-\mathrm{MgF}_{2}-\mathrm{Ag}$ periodic stack. For the set of geometrical parameters defined in Fig. 1, the structure offers negative refraction at near-infrared wavelengths $(1.4<\lambda<2 \mu \mathrm{m})$ with low losses, ${ }^{13}$ an achievement frequently interpreted as resulting from the realization of a MM with both negative permeability and permittivity.

Light scattering at a single interface between a homogeneous half-space and a 3D periodic half-space, see Fig. 1(a), represents a generalization of the textbook case of scattering at a flat interface between two homogeneous media. The calculation of the generalized Fresnel coefficients requires a correct handling of outgoing wave conditions in a semiinfinite periodic medium. Only a few numerical methods are presently available to calculate those coefficients and only two-dimensional geometries have been considered so far, mostly with dielectric materials. ${ }^{10,14,15}$ Hereafter, we calculate the scattering coefficients by using a 3D fully-vectorial formalism that is an extension of a previous work on periodic waveguides ${ }^{16}$ to structures that are periodic in both transverse $(x, y)$ directions. For the sake of consistency, we (a)

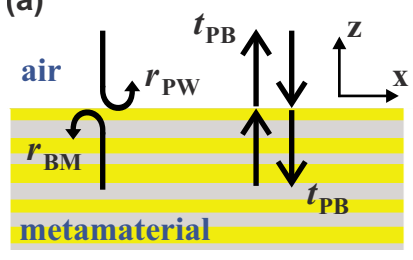

(b)

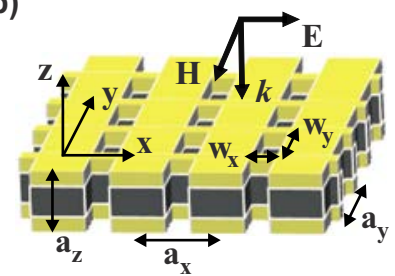

FIG. 1. (Color online) Scattering at a single interface between air and a bulk MM. (a) Definition of the main scattering coefficients: $r_{\mathrm{PW}}$ is the specular reflection of the plane wave in air, $r_{\mathrm{BM}}$ is the reflection of the fundamental Bloch mode, and $t_{\mathrm{PB}}$ is the transmission from the fundamental Bloch mode to the plane wave and vice-versa. (b) One fishnet period in $z$-direction. A square lattice $\left(\mathrm{a}_{x}=\mathrm{a}_{y}=860 \mathrm{~nm}\right)$ of rectangular holes $\left(\mathrm{w}_{x}=295 \mathrm{~nm}\right.$ and $\mathrm{w}_{y}$ $=595 \mathrm{~nm})$ is etched into an $\operatorname{Ag}(15 \mathrm{~nm})-\mathrm{MgF}_{2}(50 \mathrm{~nm})-\operatorname{Ag}(15 \mathrm{~nm})$ periodic stack $\left(\mathrm{a}_{z}=80 \mathrm{~nm}\right)$. 

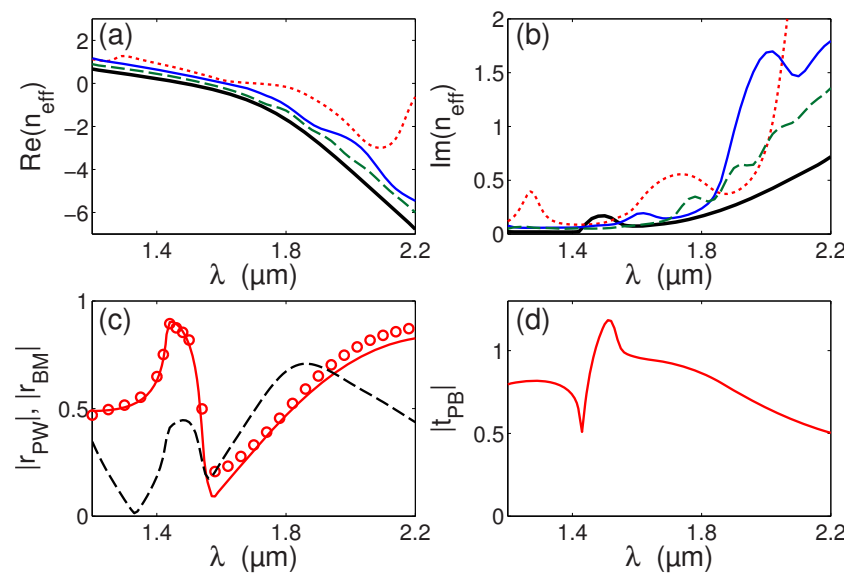

FIG. 2. (Color online) Effective index of the fundamental Bloch mode and scattering coefficients at the air/fishnet interface. (a) Real part. (b) Imaginary part. In (a) and (b) the Bloch-mode effective index (thick-solid curves) is compared with the effective refractive index retrieved with the S-parameter method $^{4}$ for several fishnet thicknesses, $d=3 \mathrm{a}_{z}$ (dotted curve), 5a $\mathrm{a}_{z}$ (solid curve) and $10 \mathrm{a}_{z}$ (dashed curve). (c) Solid curve, $\left|r_{\mathrm{PW}}\right|$, circles, $\left|r_{\mathrm{BM}}\right|$, and dashed curve, $\left|r_{\mathrm{PW}}+r_{\mathrm{BM}}\right|$. (d) Solid curve, $\left|t_{\mathrm{PB}}\right|$.

concisely recall the main lines of the approach; more details can be found in Ref. 16 .

In the first step, we determine the complete set of MM Bloch modes for a given in-plane wave vector $\left(k_{x}, k_{y}\right)$ and at a given frequency by calculating the S-matrix relating the field amplitudes in two planes separated by a single MM period $a_{z}$. The calculation is performed with the rigorous coupled-wave analysis (RCWA). ${ }^{17}$ Then the Bloch modes and their propagation constants $k_{z, \mathrm{BM}}$ are obtained by solving for a generalized eigenmode problem. This procedure removes potential numerical instabilities, ${ }^{18}$ even if a large number of Fourier harmonics is retained for the calculation or if thick and/or metallic layers are considered. The last step consists in matching the tangential field components at the air/MM interface to derive the associated scattering matrix, i.e., transmission and reflection coefficients of all Bloch modes and plane waves. For that purpose, we use a Bloch mode and a plane wave (or Rayleigh) expansion in the MM and in the upper uniform half-space, respectively.

For a MM slab to be reasonably considered as a homogeneous effective medium, the energy transfer through the slab has to be mediated dominantly by a single Bloch mode that is bouncing between the two interfaces, like a single plane wave is bouncing forth and back in a homogeneous film. ${ }^{8-10,19}$ This Bloch mode will be referred to as the fundamental Bloch mode hereafter. It can be identified from the infinite set of Bloch modes by considering either its effective index, $n_{\mathrm{BM}}=k_{z, \mathrm{BM}} / k_{0}$ with $k_{0}=2 \pi / \lambda$, which possesses one of the smallest imaginary part, or its field distribution. For the fishnet, the field distribution inside the holes much resembles that of the fundamental $\mathrm{TE}_{10}$ mode of an infinite rectangular air-waveguide in silver. The real and imaginary parts of $n_{\mathrm{BM}}$ are shown in Figs. 2(a) and 2(b). The calculation has been performed at normal incidence $\left(k_{x}=k_{y}=0\right)$. The refractive index of the $\mathrm{MgF}_{2}$ films is $n=1.38$ and the frequencydependent permittivity of silver is taken from Ref. 20. A total number of $N=\left(2 m_{x}+1\right)\left(2 m_{y}+1\right)=1681$ Fourier harmonics has been used in the calculation, $m_{x}=m_{y}=20$ being the truncation ranks in both transverse directions. In agreement with the experimental and theoretical data in Ref. 13, it is found
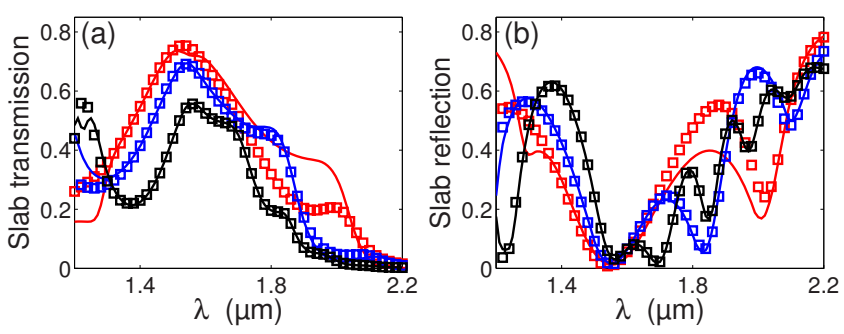

FIG. 3. (Color online) Transmission (a) and reflection (b) efficiencies of fishnet slabs with 3 (red), 5 (blue), and 10 (black) unit cells for normal incidence. Fully-vectorial RCWA data are shown by solid curves, and results obtained by the single-Bloch-mode approximation from Eqs. (1a) and (1b) are indicated by squares.

that $\operatorname{Re}\left(n_{\mathrm{BM}}\right)<0$ for $\lambda>1.5 \mu \mathrm{m}$ and that the Bloch mode attenuation is remarkably weak, $\operatorname{Im}\left(n_{\mathrm{BM}}\right)<0.18$ for $1.5<\lambda$ $<1.8 \mu \mathrm{m}$.

At a single interface, the coupling between the zerothorder plane wave and the fundamental Bloch mode defines three independent scattering coefficients, the specular reflection of the plane wave in air, $r_{\mathrm{PW}}$, the reflection of the fundamental Bloch mode inside the $\mathrm{MM}, r_{\mathrm{BM}}$, and the transmission between the fundamental Bloch mode and the plane wave, $t_{\mathrm{PB}}$, see Fig. 1(a). Reciprocity imposes that the latter is equal to the transmission coefficient between the incident plane wave and the fundamental Bloch mode. The moduli of the scattering coefficients are shown by red curves in Figs. 2(c) and 2(d) for the spectral range of interest and for normal incidence. Several interesting features are observed. First, we note that the spectra of every scattering coefficient exhibit a resonance for $1.4<\lambda<1.6 \mu \mathrm{m}$. This can be understood as the signature of the excitation of a surface mode at the air/ fishnet interface. Second, after entering the negative-index regime $(\lambda>1.5 \mu \mathrm{m})$, every scattering coefficient smoothly varies with the wavelength, attesting that the negative index is not due to any acute resonance. Finally, it is interesting to check whether the calculated reflection coefficients satisfy the usual Fresnel relation at an interface between two homogeneous media, $r_{\mathrm{PW}}=-r_{\mathrm{BM}}$. The moduli of the scattering coefficients are almost equal over the full spectral range $\left(\left|r_{\mathrm{PW}}\right| \approx\left|r_{\mathrm{BM}}\right|\right)$ but the phases of the coefficients are not opposite [see Fig. 2(c)]. This shows that the air/MM interface cannot be fully understood as an interface between two uniform media.

The single interface scattering coefficients being determined, we now test the single-Bloch-mode approximation for a fishnet slab. Under the assumption that the energy transport is mediated only by the fundamental Bloch mode, the reflection $r$ and transmission $t$ of a fishnet slab of thickness $d$ are given by

$$
\begin{aligned}
& r=r_{\mathrm{PW}}+\left(r_{\mathrm{BM}} t_{\mathrm{PB}}{ }^{2} u^{2}\right) /\left(1-r_{\mathrm{BM}}{ }^{2} u^{2}\right), \\
& t=\left(t_{\mathrm{PB}}{ }^{2} u\right) /\left(1-r_{\mathrm{BM}}{ }^{2} u^{2}\right),
\end{aligned}
$$

with $u=\exp \left(\mathrm{i} k_{0} n_{\mathrm{BM}} d\right)$. Note that these Fabry-Perot equations hold for any Bloch mode, propagating or damped, which is encountered in tunneling transmissions. Figure 3 compares the specular transmission and reflection efficiencies (solid curves) calculated with the $\mathrm{RCWA}^{17}$ for three slab thicknesses, $d=3,5$, and $10 a_{z}$, with those (squares) obtained from Eqs. (1a) and (1b). The very good agreement observed leads us to the conclusion that, for this fishnet geometry, the 

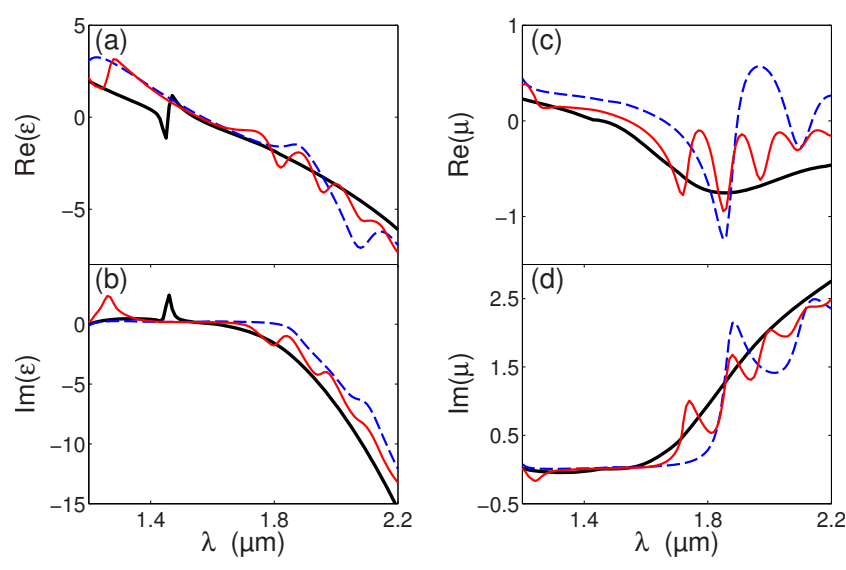

FIG. 4. (Color online) Retrieved effective permittivities, $\varepsilon_{\text {eff }}$, and permeabilities, $\mu_{\text {eff. }}$ The dashed and thin solid curves represent the retrieved parameters obtained with the S-parameter method ${ }^{4}$ using the RCWA data obtained for $d=5$ and $10 \mathrm{a}_{z}$, respectively. The thick-solid curve is obtained by solving Eqs. (2) and (3).

light propagation may be mainly described by a single dominant Bloch mode.

It is instructive to consider what the single interface problem teaches us on the homogenization problem. For that purpose, let us apply the S-parameter retrieval algorithm ${ }^{4}$ to the RCWA data obtained for the scattering coefficients of the finite-thickness slab. The effective permittivity $\varepsilon_{\text {eff }}$ and permeability $\mu_{\text {eff }}$ are retrieved for three slab thicknesses and the retrieved effective indices, $n_{\text {eff }}= \pm\left(\varepsilon_{\text {eff }} \mu_{\text {eff }}\right)^{1 / 2}$, are compared with the dominant-Bloch-mode effective index $n_{\mathrm{BM}}$ in Figs. 2(a) and 2(b). An overall qualitative agreement is achieved. However, there are also some discrepancies, especially for large wavelengths, where one observes a spurious oscillatory behavior for $n_{\mathrm{eff}}$, with minima or maxima that depends on the fishnet thickness. Let us emphasize that since all computations are performed with the same number of Fourier harmonics, the discrepancy between $n_{\text {eff }}$ and $n_{\mathrm{BM}}$ is not due to any numerical artifact. It is rather unexpected, especially for thick slabs $\left(d=5\right.$ and $\left.10 a_{z}\right)$, for which the single-Blochmode approximation is highly accurate (Fig. 3). As explained before, the reason for this discrepancy comes from the fact that the scattering properties at the air/MM interface differ from those between two uniform media, in particular, $r_{\mathrm{PW}}$ $\neq-r_{\mathrm{BM}}$.

In our opinion, only MMs that satisfy the single-Blochmode approximation may be homogenized and any homogenization method should guarantee that the retrieved parameters, $\varepsilon_{\text {eff }}$ and $\mu_{\text {eff }}$, satisfy

$$
n_{\mathrm{BM}}=\left(\varepsilon_{\mathrm{eff}} \mu_{\mathrm{eff}}\right)^{1 / 2}
$$

at least with a good approximation. If not, the effective parameters necessarily depend on the slab thickness. Equation (2) alone does not allow to fully determine the effective parameters. In addition, we propose to use the Fresnel relation for the reflection between two homogeneous media as a function of the impedance. For normal incidence, $z_{\text {eff }}$ $=\left(\mu_{\text {eff }} / \varepsilon_{\text {eff }}\right)^{1 / 2}$ and one gets

$$
\left(\mu_{\mathrm{eff}} / \varepsilon_{\mathrm{eff}}\right)^{1 / 2}=\left(1+r_{\mathrm{PW}}\right) /\left(1-r_{\mathrm{PW}}\right) .
$$

Equations (2) and (3) can be directly solved for $\varepsilon_{\text {eff }}$ and $\mu_{\mathrm{eff}}$, without any ambiguity due to the square root. Figure 4 com- pares the corresponding effective parameters with those obtained from a finite-thickness slab with the S-parameter retrieval algorithm. ${ }^{4}$ Again, the unphysical oscillatory behavior is fully removed with the present approach. Formally, this procedure can be extended toward oblique incidence. The retrieved parameters $\varepsilon_{\text {eff }}$ and $\mu_{\text {eff }}$ will then depend on the angle of incidence as a result of spatial dispersion due to the mesoscopic nature of MMs at optical frequencies. ${ }^{21}$ In this case, description of light propagation requires particular care to be shown elsewhere. In practice, the proposed retrieval procedure based on Eqs. (2) and (3) can be applied to experimental data for thick MMs. In this limit, $|u| \ll 1$ and the scattering coefficients of Eqs. (1a) and (1b) become $r=r_{\mathrm{PW}}$ and $t=t_{\mathrm{PB}}{ }^{2} u ; r_{\mathrm{PW}}$ is thus directly measured and $n_{\mathrm{BM}}$ can be indirectly (but unambiguously) determined by measuring the transmission coefficients of two MM slabs with different thicknesses for instance.

In conclusion, we have studied the scattering at an air/MM interface. We have shown that the knowledge of the single-interface scattering coefficients allows for an in-depth test of the homogenization validity. Moreover, we have proposed an innovative approach for deriving the effective parameters. The method emphasizes the key role played by the fundamental Bloch mode and provides retrieved parameters that are more accurate or stable than those obtained by general methods based on far-field physical quantities.

The authors acknowledge financial support from the bilateral French-German program PROCOPE under Grant Nos. 21956SD and 50076916.

${ }^{1}$ R. A. Shelby, D. R. Smith, and S. Schultz, Science 292, 77 (2001).

${ }^{2}$ V. M. Shalaev, Nat. Photonics 1, 41 (2007).

${ }^{3}$ C. M. Soukoulis, S. Linden, and M. Wegener, Science 315, 47 (2007).

${ }^{4}$ D. R. Smith, S. Schultz, P. Markos, and C. M. Soukoulis, Phys. Rev. B 65 , 195104 (2002).

${ }^{5}$ T. Koschny, P. Markoš, E. N. Economou, D. R. Smith, D. C. Vier, and C. M. Soukoulis, Phys. Rev. B 71, 245105 (2005).

${ }^{6}$ D. Seetharamdoo, R. Sauleau, K. Mahdjoubi, and A. C. Tarot, J. Appl. Phys. 98, 063505 (2005).

${ }^{7}$ D. R. Smith and J. B. Pendry, J. Opt. Soc. Am. B 23, 391 (2006).

${ }^{8}$ S. Zhang, W. Fan, N. C. Panoiu, K. J. Malloy, R. M. Osgood, and S. R. J. Brueck, Opt. Express 14, 6778 (2006).

${ }^{9}$ C. Rockstuhl, C. Menzel, T. Paul, T. Pertsch, and F. Lederer, Phys. Rev. B 78, 155102 (2008).

${ }^{10}$ W. Śmigaj and B. Gralak, Phys. Rev. B 77, 235445 (2008).

${ }^{11}$ J. Petschulat, C. Menzel, A. Chipouline, C. Rockstuhl, A. Tünnermann, F. Lederer, and T. Pertsch, Phys. Rev. A 78, 043811 (2008).

${ }^{12}$ K. Vynck, D. Felbacq, E. Centeno, A. I. Căbuz, D. Cassagne, and B. Guizal, Phys. Rev. Lett. 102, 133901 (2009).

${ }^{13}$ J. Valentine, S. Zhang, T. Zentgraf, E. Ulin-Avila, D. Genov, G. Bartal, and X. Zhang, Nature (London) 455, 376 (2008).

${ }^{14}$ K. Dossou, M. A. Byrne, and L. C. Botten, J. Comput. Phys. 219, 120 (2006).

${ }^{15}$ A. Fallahi and C. Hafner, J. Opt. Soc. Am. A 27, 40 (2010).

${ }^{16}$ G. Lecamp, J. P. Hugonin, and P. Lalanne, Opt. Express 15, 11042 (2007).

${ }^{17}$ L. Li, J. Opt. Soc. Am. A 14, 2758 (1997).

${ }^{18}$ Q. Cao, P. Lalanne, and J. P. Hugonin, J. Opt. Soc. Am. A 19, 335 (2002).

${ }^{19}$ P. Lalanne, S. Astilean, P. Chavel, E. Cambril, and H. Launois, Opt. Lett. 23, 1081 (1998).

${ }^{20}$ E. D. Palik, Handbook of Optical Constants of Solids (Academic, New York, 1985).

${ }^{21}$ C. Menzel, T. Paul, C. Rockstuhl, T. Pertsch, S. Tretyakov, and F. Lederer, Phys. Rev. B 81, 035320 (2010). 